\title{
Art. XXIV DEL GATT-OMC: LA RELACIÓN ENTRE REGIONALISMO Y MULTILATERALISMO
}

\author{
Germán A. de la Reza*
}

Fecha de recepción: 01 de agosto de 2014. Fecha de aceptación: 03 de diciembre de 2014.

\begin{abstract}
RESUMEN
En el presente trabajo estudiamos la interacción entre el regionalismo y el multilateralismo a través del Art. XXIV del GATT-OMC y en un plano más general el grado de conexión que existe entre las dinámicas regionales y las fuerzas globales tomando en consideración los factores organizacionales. Los resultados de la investigación sugieren que el multilateralismo y el regionalismo responden a intereses sistémicos relativamente autónomos, donde uno representa una alternativa secundaria a los beneficios y necesidades del otro.
\end{abstract}

Palabras clave: regionalismo, multilateralismo, acuerdos regionales, comercio mundial, libre comercio.

Clasificación JEL: F13, F53, F55, O19.

\section{Art. XXIV of THE GatT/WTO: THE RelationshiP BETWEEN REgionalism and Multilateralism}

\begin{abstract}
This work studies the interaction between regionalism and multilateralism by examining Art. XXIV of the GATT/WTO and, more generally the degree to which regional dynamics and global forces are connected, taking into account organizational factors. The results of the research suggest that multilateralism and regionalism respond to relatively autonomous systemic interests, where one is a secondary alternative to the benefits and needs of the other.
\end{abstract}

Key Words: Regionalism, multilateralism, regional agreements, world trade, free trade.

\footnotetext{
* Universidad Autónoma Metropolitana, unidad Xochimilco, México. Correo electrónico: delareza@hotmail.com
} 


\section{ART. XXIV DU GATT-OMC: LE RAPPORT ENTRE RÉGIONALISME ET MULTILATÉ- RALISME \\ Résumé}

Dans ce travail, nous étudions l'interaction entre le régionalisme et le multilatéralisme à travers l'Art. XXIV du GATT-OMC et, sur un plan plus général, le degré de relation qui existe entre les dynamiques régionales et les forces globales, en prenant en compte les facteurs organisationnels. Les résultats de la recherche suggèrent que le multilatéralisme et le régionalisme répondent à des intérêts systémiques relativement autonomes, où l'un représente une alternative secondaire aux bénéfices et aux besoins de l'autre. Mots clés: régionalisme, multilatéralisme, accords régionaux, commerce mondial, libre commerce

\section{ART. XXIV DO GATT-OMC: A RELAÇÃO ENTRE REGIONALISMO E MULTILATE- RALISMO}

Resumo

No presente trabalho estudamos a interação entre o regionalismo e o multilateralismo através do Art. XXIV do GATT-OMC e num plano mais geral o grau de conexão que existe entre as dinâmicas regionais e as forças globais tomando em consideraçáo os fatores organizacionais. Os resultados da pesquisa sugerem que o multilateralismo e o regionalismo respondem a interesses sistêmicos relativamente autônomos, onde um representa uma alternativa secundária aos benefícios e necessidades do outro.

Palavras-chave: regionalismo, multilateralismo, acordos regionais, comércio mundial, livre comércio.

关贸总协定-世贸组织的第 24 条款：地区主义与多边主义的关系

摘要:

本文通过分析关贸总协定/世贸组织中的第 24 条款研究了地区主义与多边主义之间 的关系, 特别是在考虑到组织的条件下, 分析了地区动力与全球力量在多大程度上 是相互联系的。研究结果表明, 多边主义与地区主义在自主利益体系内存在互动关 系，在利益方面，互为替代选择，并互相需要。

关键词：地区主义多边主义地区安排世界贸易自由贸易 


\section{INTRODUCCIÓN}

La relación entre regionalismo y multilateralismo vuelve a estar en la mira de analistas y tomadores de decisiones después de una larga y nutrida controversia. La razón de este renovado interés lo constituye el contraste entre dos procesos: el creciente número de notificaciones de Acuerdos Comerciales Regionales (ACR) y el estancamiento de las negociaciones multilaterales. El instrumento clave para su conexión, el Art. XXIV de la Organización Mundial de Comercio (OMC), prevé que las uniones aduaneras y las áreas de libre comercio no tengan una protección externa mayor ni más restrictiva que la incidencia general anterior a su creación. Desde 1994, agrega a sus estipulaciones un plazo de desgravamen limitado a 10 ańos y la compatibilización con el multilateralismo siguiendo criterios más estrictos. El objetivo es limitar el recurso a los ACR y robustecer los compromisos globales.

Sin embargo, de 1994 a 2014 el número de acuerdos regionales creció de manera exponencial y hoy en día ampara más de la mitad del comercio mundial. En junio del último año, estaban vigentes 248 uniones aduaneras, zonas de libre comercio y acuerdos preferenciales, mientras que en los 47 años correspondientes al periodo de existencia del Acuerdo General sobre Aranceles Aduaneros y Comercio (GATT), solo se notificaron 124, exactamente la mitad. En paralelo, la agenda multilateral registraba una evolución relativamente accidentada en virtud del fracaso en las negociaciones agrícolas en 2003 y, sobre todo, de la crisis financiera global. No es el único elemento de ese contaste. El aumento de los ACR lo es en parte de los acuerdos Norte-Sur no obstante ser más costosos y más difíciles de vender políticamente, al no incluir la cláusula de no reciprocidad como compensación a las desventajas del menor desarrollo. Otros grupos de acuerdos tienden al bilateralismo o buscan trascender las regiones (oMC, 2012: 187).

En el presente trabajo estudiamos la interacción entre el regionalismo y el multilateralismo a través del Art. XXIV del GATT-OMC; en un plano más general, la conexión entre las dinámicas regionales y las fuerzas globales tomando en consideración sus factores organizacionales. En la primera sección reseñamos las principales hipótesis de la dicotomía regionalismo-multilateralismo; en la segunda analizamos el Art. XXIV y sus refuerzos, mientras que en la tercera pasamos revista a la situación normativa de los ACR. En las secciones cuarta y quinta evaluamos los efectos del Art. XXIV considerando dos variables conectadas con sus expectativas: la dinámica de creación de los ACR y el examen de compatibilidad. Los resultados de la investigación sugieren que el multilateralismo y el regionalismo responden a intereses sistémicos relativamente autónomos, donde uno representa una alternativa secundaria a los beneficios y necesidades del otro. 


\section{RIVALES, AMIGOS O COPARTÍCIPES}

La mayoría de los estudios sobre los vínculos entre el multilateralismo y el regionalismo sostienen que los ACR representan una opción second best frente al óptimo de la liberalización global. Las posiciones más críticas al regionalismo interpretan esta estrategia como un esquema intrínsecamente discriminador (Bhagwati, 1993) capaz de poner en riesgo el futuro del sistema multilateral (Bhagwati y Panagariya, 1996). Para paliar esos efectos, algunos autores recomiendan que las preferencias regionales se extiendan al resto de los miembros de la OMC después de un cierto periodo de tiempo (Lee, 2011), lo que equivale en los hechos a programar su desaparición, una opción poco realista a juzgar por la buena salud de las iniciativas regionales. Otra medida es que los compromisos multilaterales alcancen los mismos estándares de la integración regional a fin de ejercer un sentido convergente sobre los factores que cimentan la omc. Sin embargo, esa política podría tener el efecto contrario y estimular la creación o desarrollo de las iniciativas regionales (Fabbricotti, 2009).

Otro grupo de conjeturas sostiene que el regionalismo colabora con el multilateralismo en la realización de objetivos comunes. Según este enfoque, el regionalismo representa "un paso decisivo hacia el fortalecimiento del multilateralismo" (UNASUR, 2008), una relación indirecta, ya que los factores que compelen a la integración regional a ser multilateralmente compatible representan un fin en sí mismos. Entre ellos cabe citar: competitividad global, globalización de los mercados financieros, incremento de la demanda de consumidores y mayor movilidad e innovación tecnológica (Mistry, 1995). Una idea que comparten otros autores es que el regionalismo facilita las negociaciones en áreas que requieren elevados niveles de coordinación, lo cual permite profundizar la cobertura temática y preparar la agenda multilateral (Krugman, 1993; Sampson, 1996; López y Soler, 1998).

La visión intermedia destaca que el regionalismo y el multilateralismo constituyen instancias rivales, pero no exclusivas, ya que el resultado es la complementariedad (Michalak y Gibb, 1997). Aquí se defiende la idea de que los ACR no debilitan el sistema multilateral, sino que operan como building blocks de la liberalización no discriminatoria (Herz y Waner, 2011). Para uno de los estudios que tienen en la mira el tamaño de las economías, el regionalismo representa un incentivo multilateralista si fortalece la capacidad negociadora de los pequeños países (Nordstrom, 1995). Para otro, las áreas de libre comercio entre economías grandes apoyan los avances en materia de arancel NMF y las compuestas de economías pequeñas, los perjudican (Nomura et al., 2013). 
Una conjetura particular sobre el comercio de servicios, afirma que "no hay dilema entre el escenario multilateral o regional", ya que "los países se sienten a sus anchas con el hecho de que coexistan ambos sistemas" (Marconini, 2010: 92). Esto se debe a que los ACR pueden no ocasionar perjuicios a terceros países (Pant y Sadhukhan, 2009). Dos hipótesis condicionales tocan el problema de la competitividad. En un caso se dice que los ACR facilitan el multilateralismo si las ventajas comparativas internas y externas a la región son similares (Kono, 2007); en el otro, que estos acuerdos promueven el comercio internacional salvo cuando contienen restricciones (Chen y Mattoo, 2008). Más allá de las diferencias de posiciones, la mayoría de autores recomienda multilateralizar al regionalismo como recurso ante los "riesgos de discriminación” y de afectación al sistema mundial (Baldwin et al., 2009).

Las hipótesis finales de esta sección apelan a elementos de coyuntura para explicar la multiplicación de los ACR. Según Baldwin (1995), ese fenómeno se genera en América y Europa gracias a "dos eventos idiosincráticos". En el Hemisferio Occidental, el tratado de México con Estados Unidos y Canadá rompió con el esquema tradicional de relacionamiento interamericano y junto con otras iniciativas, provocó un efecto dominó sobre la creación de los ACR, principalmente bilaterales. En el viejo continente, el evento produjo el mismo efecto fue la compleción del mercado único.

Menor presencia en esta controversia tiene la posición según la cual el regionalismo se desarrolla de manera relativamente autónoma respecto de los impulsos multilaterales. ${ }^{2}$ Esta hipótesis no ignora la existencia de canales de comunicación ni el efecto de vasos comunicantes o la equivalencia de los compromisos regional y multilateral. Su empleo en el presente estudio pretende explicar la falta de correlación entre ambos niveles y la falta de efectividad del Art. XXIV. La principal implicación teórica de esta hipótesis es que el regionalismo busca mantener su vitalidad como opción de política económica mediante una mayor cobertura temática y la profundización de los compromisos (Kerry, 2007: 199). En ese camino puede rivalizar, colaborar o complementar las tareas de la OMC, aunque esencialmente es un colaborador en la construcción de la arquitectura económica internacional. estimulado por acuerdos de libre comercio "defensivos", i.e., creados con el fin de reducir la discriminación creada por otros acuerdos.

2 La hipótesis de la autonomía sistémica del regionalismo tiene adeptos sobre todo en las Relaciones Internacionales: Buzan y Waever (2003: 18); Hoogensen (2005: 269); Kerry (2007: 199, ss.). Para una defensa desde posiciones de la nueva economía política, véase Hettne y Söderbaum (2000). 


\section{EL ART. XXIV Y SUS REFUERZOS}

Durante la negociación de la Ronda Uruguay, los representantes de los países miembros observaron que ninguno de los ACR notificados al GATT desde 1947 había sido censurado por su inconsistencia. Para corregir ese hecho, atribuido a la excesiva flexibilidad de la norma, en 1994 se aprueba el Entendimiento relativo a la interpretación del artículo XXIV, el cual busca determinar la consistencia de los derechos aduaneros regionales mediante "la evaluación general de las tasas de las tarifas medias ponderadas y de los derechos de aduanas recaudados". Reconoce como medida básica las tasas de los derechos, aunque la omc obtiene la facultad de solicitar información adicional sobre las medidas, las reglamentaciones, la cobertura de productos y los flujos comerciales particulares. El plazo de desgravación, que anteriormente debía ser "razonable", ahora se recomienda que no debe rebasar los 10 años, "salvo en casos excepcionales".

El Entendimiento completa su acción con tres directivas: 1) las contradicciones en la interpretación de la normativa deben resolverse apelando a las decisiones vinculantes del Mecanismo de Solución de Controversias (DSU, por sus siglas inglesas); ${ }^{3}$ 2) los acuerdos relativos al comercio de servicios se examinan con base en el Art. V del Acuerdo General sobre Comercio de Servicios (GATS), el cual estipula condiciones similares al Art. XXIV, pero en su disciplina; y 3) las partes contratantes se comprometen a revisar periódicamente los acuerdos para identificar contradicciones o violaciones, apelando para ello al Comité de Acuerdos Comerciales Regionales (CACR), el que ha recibido el mandato de examinar los acuerdos y presentar sus informes y las

Según el párrafo 12 del documento, "Podrá recurrirse a las disposiciones de los artículos XXII y XXIII del GATT de 1994, desarrolladas y aplicadas en virtud del Entendimiento sobre Solución de Diferencias, con respecto a cualesquiera cuestiones derivadas de la aplicación de las disposiciones del Artículo XXIv referentes a uniones aduaneras, zonas de libre comercio o acuerdos provisionales tendientes al establecimiento de una unión aduanera o de una zona de libre comercio". En lenguaje críptico, este párrafo quiere decir que los problemas de interpretación sobre la posible inconsistencia de los acuerdos se resolverán de manera inapelable.

El Art. V del Gats incluye las siguientes normas: 1) Los países podrán participar en acuerdos regionales si la cobertura sectorial es substancial, considerada en función del número de sectores, el volumen del comercio afectado y los modos de suministro. 2) Se elimina en lo esencial, toda discriminación entre las partes. 3) Prever la flexibilidad de la norma en el caso de los países en desarrollo, tanto en general como en el caso de los sectores y subsectores. 4) No elevar el nivel global de obstáculos al comercio de servicios dentro de los respectivos sectores o subsectores respecto al nivel preexistente. 
oportunas recomendaciones. Entre los asuntos que debe tratar en sus informes figuran las consecuencias de los acuerdos sobre el sistema multilateral (OMC, 1996).

Estas medidas e instrumentos, cuya lógica descansa claramente en la economía política de la liberalización del comercio (Pant y Sadhukhan, 2009: 223), son revisados de manera periódica por la oMC, pero todavía no se logran los resultados esperados. En noviembre de 2001, la Cuarta Conferencia Ministerial celebrada en Doha testimonia el balance insatisfactorio e insiste en la importancia de avanzar en la compatibilidad de los acuerdos. Ese año China se convierte en miembro de la OMC, una adhesión que parece reforzar el sistema multilateral por la importancia económica del país, en ese entonces $7^{\circ}$ exportador y $8^{\circ}$ importador de bienes del mundo (omc, 2001). Tiempo después, empero, la ausencia de consenso en la Reunión Ministerial de Cancún en septiembre de 2003 impide el progreso en materia de acceso a mercados y de normas comerciales, dejando en entredicho no sólo el futuro de las reformas de la OMC, sino el abandono de iniciativas como el Área de Libre Comercio de las Américas (ALCA), cuyas comisiones negociadoras habían vinculado el capítulo agrícola a los resultados de la omc.

La Conferencia de Hong Kong realizada en noviembre de 2005 y la Declaración Ministerial de diciembre de ese año, no logran sortear los escollos del multilateralismo y la Cumbre de las Américas de Mar de la Plata, realizada casi al mismo tiempo, deja traslucir el agotamiento de las negociaciones agrícolas y de los intentos por revivir el ALCA. El ingreso de Rusia, Samoa y Montenegro en diciembre de 2011 y las expectativas con las que se organiza la Novena Conferencia Ministerial en Bali en diciembre de 2013, no pueden evitar ser condicionados por el periodo de crisis mundial. ${ }^{5}$ Respecto de la proliferación de ACR, la OMC hace un llamado a actuar para que la integración regional complemente, no amenace al multilateralismo de comercio. Aunque reconoce la mayor cobertura de una parte de los acuerdos regionales, asegura que la cobertura bilateral de otros acuerdos provoca "muy a menudo [que] se solapen, lo que da lugar a un régimen cada vez más complejo de reglamentaciones comerciales diferentes" (OMC, 2013).

En la actualidad, la OMC mantiene su propósito de crear una zona de libre comercio multilateral alrededor del año 2020, aunque las secuelas de la crisis financiera global podrían postergar su creación y ajustar sus aspiraciones a las

Discurso de agradecimiento de la presidente del Consejo General, Elin Johansen, al gobierno de Indonesia por su oferta de albergar la Novena Conferencia Ministerial en la isla de Bali en 2013. 
nuevas realidades del sistema internacional. En julio de 2014, la VI Cumbre del Grupo Brasil, Rusia, India y Sudáfrica (BRICs) acordó la creación de un Banco de Desarrollo y un Fondo de Reservas con un capital inicial de 50 mil millones de dólares. Aunque la agrupación no se plantea objetivos comerciales, su creciente impacto en el comercio internacional no dejará de influir en las negociaciones multilaterales del sector agrícola.

Los 21 países del Foro de Cooperación Económica Asia-Pacífico (APEC), a su vez prevén la creación de una gigantesca área de libre comercio en 2020, compuesta, entre otros países, por Estados Unidos, China, Rusia, Japón, Corea del Sur y México. ${ }^{6}$ Se trata de un proyecto de difícil concreción y que en fechas recientes ha perdido dinamismo, si bien apunta en el sentido de la reconfiguración del sistema de gobernanza internacional del cual la OMC es un componente fundamental. De estos países, China viene ampliando su liderazgo en la formación del nuevo regionalismo asiático: participa en la creación de nuevos acuerdos e instituciones, refuerza la Asociación de Naciones del SurEste Asiático (ASEAN) y favorece la exploración de nuevas vías de cooperación regional (Pempel, 2008).

Lo propio puede decirse del eventual impacto de dos mega-acuerdos en proceso de negociación: el Acuerdo Estratégico Transpacífico de Asociación Económica (TPP) y la Asociación Transatlántica para el Comercio y la Inversión (ATCI). En 2006, el TPP era un acuerdo entre economías relativamente pequeñas; luego de una veintena de rondas negociadoras y de la adhesión de países como Estados Unidos, Japón, Canadá, Australia y México, constituye una importante instancia negociadora de la profundización de los estándares de facilitación del comercio. ${ }^{8}$ La ATCI, por su parte, una iniciativa que se remonta al fracaso de la Ronda de Doha, pretende instituir un área de libre co-

Los restantes integrantes del APEC son: Australia, Brunei, Canadá, Indonesia, Malasia, Nueva Zelanda, Filipinas, Singapur, Tailandia, China Taipéi, Hong Kong, Papúa Nueva Guinea, Chile, Perú y Vietnam.

El nuevo regionalismo asiático surge como reacción a las condiciones de la crisis financiera de 1997-1998. Una de las medidas que buscan la interdependencia de 16 países de la región, el refuerzo comercio intrarregional, frecuentemente tiene a China como país hub. Sus redes han elevado la participación del intercambio regional en el total a niveles superiores a los que tenían Europa y Norteamérica antes de sus respectivos esfuerzos de integración (ADB, 2008: 40 y ss.).

Los otros miembros del tpp son: Brunei, Chile, Malasia, Nueva Zelanda, Perú, Singapur y Vietnam. Obsérvese que los tres países latinoamericanos del Tpp, además de Colombia, conforman a partir de junio de 2012 la llamada Alianza del Pacífico, un esquema con objetivos en proceso de definición. 
mercio que abarque a Estados Unidos y a la Unión Europea. Se espera que las ganancias económicas anuales para Estados Unidos sean de 95 mil millones de euros, para la UE de 119 mil millones de euros y para el resto del mundo de 100 mil millones de euros (Francois et al., 2013: vii). En junio de 2013, la Comisión Europea giró sus instrucciones para la negociación de este acuerdo, el cual se espera que esté listo en $2015^{9}$

\section{SITUACIÓN DE LOS ACR RESPECTO DEL ART. XXIV}

De acuerdo con la nomenclatura de la OMC, los ACR se dividen en tres categorías: zonas de libre comercio, uniones aduaneras y arreglos de alcance parcial (aunque tengan coberturas plurilaterales o bilaterales, reciben la denominación de acuerdos regionales). Casi la totalidad de los más recientes han sido legalizados ante la OMC apelando a una de sus tres normas: ${ }^{10}$ el Art. XXIV, la Cláusula de Habilitación y el Art. V del Gats, en el último caso cuando se busca amparar el comercio de servicios. ${ }^{11}$ Esto explica por qué el número total de notificaciones y de los ACR reales no sea el mismo: si un acuerdo recubre los comercios de bienes y de servicios, debe realizar dos notificaciones separadas.

Aparte de su número, los ACR vigentes destacan por varias características. Un primer rasgo es la preponderancia de las zonas de libre comercio en detrimento de otras formas de integración. Las uniones aduaneras ocupan un lugar

El texto de las instrucciones de la Comisión Europea fueron desclasificadas en octubre de 2014. Para consultar su contenido, véase http://data.consilium.europa.eu/doc/document/ ST-11103-2013-DCL-1/en/pdf

La lista de los ACR mencionados en las presentaciones fácticas publicadas antes del 10 de junio de 2014, incluían a 55 acuerdos no notificados, la mayoría de ellos con varios ańos de existencia, 25 realizados exclusivamente entre miembros de la ALADI (OMC, 2014b).

La iniciativa al origen del GATT y del Art. XXIV, da prioridad a los esquemas de unión aduanera y zona de libre comercio sobre las concesiones no recíprocas, debido a que los primeros "crean anchas zonas de comercio, levantan los obstáculos a la competitividad, hacen posible una mejor asignación de recursos y elevan el bienestar", mientras que el sistema preferencial "obstruye la producción y restringe el incremento de los ingresos y la demanda" (Wilcox, 1949: 70-71). Poco después, Viner (1950: 48) refuta esa presunción por considerar que los costos del sistema preferencial "pueden ser imputados, al menos potencialmente, a las uniones aduaneras y a la inversa, ya que los beneficios de la unión aduanera también pueden darse en las uniones preferenciales si las circunstancias son las adecuadas". En la década de 1960, Mundell (1964) defiende el mismo argumento, aunque habrá que esperar hasta 1979 para que el GATT reconozca los acuerdos preferenciales. 
bastante relegado, son más longevas y asocian a un número mayor de países, lo cual se observa también en algunos acuerdos preferenciales, como la Asociación Latinoamericana de Integración (ALADI) o los acuerdos transregionales. La siguiente característica es el predominio de las coberturas bilaterales, lo que lleva a los ACR a formar sistemas hub-and-spoke, donde uno o más países centrales administran simultáneamente varias preferencias. ${ }^{12}$ A esto se asocia el creciente peso de los acuerdos Norte-Sur, compuestos de economías industrializadas y en curso de desarrollo. ${ }^{13}$ El hecho es significativo, pues estos acuerdos incluyen a economías dispares y suelen ser más costosos políticamente (Pant y Sadhukhan, 2009: 224).

Otra característica de los ACR es la búsqueda de cobertura de los mayores flujos comerciales. En la década de 1990, la recomendación que subyacía a esta política fue la de integrar a los socios "naturales", i.e., aquellos con los que se comercia en 25 por ciento o más del total (CEPAL 1994: 78). ${ }^{14}$ Con el tiempo, esta tendencia condujo a un ordenamiento de tipo estelar que concentró los ACR en pocos países. Aparte de la Unión Europea, 12 economías hub están al origen de 280 acuerdos, un promedio de 20.5 por país central. En el Hemisferio Occidental, los países hub son cuatro: Estados Unidos, México, Chile y Perú, los cuales suman entre sí 71 acuerdos (véase cuadro 1).

Obsérvese que estas características ilustran menos la expansión del comercio internacional, que la posibilidad de direccionar las preferencias comerciales. En ese sentido, indiferente de sus resultados económicos, la mayoría de los ACR parece mejor preparada para hacer frente a las crisis económicas, que las largas, inciertas e impopulares rondas multilaterales. ¿¿Cuál es el papel del Art. XXIV en este contexto?

El término fue utilizado por primera vez en la literatura económica por Lipsey y Smith (1989), Kowalczk y Wonnacott (1992), a fin de modelar los efectos económicos del bilateralismo estadounidense sobre la economía canadiense.

Esta hipótesis se acompaña de otra: los acuerdos entre pequeñas economías tienden a desviar comercio como resultado de su incapacidad de suplir todas las necesidades de importación y sus mayores costos en caso de decidirse a producir en el ámbito regional (Schiff, 1997, Schiff y Winters, 2004).

La CEPAL ha superado la concepción regionalista de esos años. En fechas recientes promueve la interacciones sistémica con el fin de acelerar el desarrollo económico. En el campo de la integración observa el "creciente peso de las economías emergentes y de las relaciones Sur-Sur en la economía mundial", así como "la búsqueda de una mayor cooperación regional y subregional" (CEPAL, 2014: 348). 
Cuadro 1. Países hub y notificaciones a la OMC

\begin{tabular}{lccc}
\hline & Art. XXIV & Art. V & ACR \\
\hline Unión Europea & 33 & 10 & 34 \\
Norvega & 25 & 9 & 26 \\
Suiza & 26 & 9 & 26 \\
Islandia & 25 & 10 & 26 \\
Chile & 24 & 18 & 24 \\
Turquía & 20 & 0 & 20 \\
Singapur & 20 & 16 & 20 \\
México & 17 & 13 & 17 \\
Rusia & 16 & 0 & 16 \\
Ucrania & 16 & 0 & 16 \\
Perú & 16 & 11 & 16 \\
India & 15 & 4 & 15 \\
Estados Unidos & 14 & 13 & 14 \\
\hline
\end{tabular}

Fuente: OMC, ITC Y UNCTAD (2013: 181-182).

\section{¿CORRELACIÓN ENTRE LOS PROCESOS REGIONAL Y MULTILATERAL?}

Los efectos de la acción del Art. XXIV y sus refuerzos son diversos, no lineales y difícilmente cuantificables. Para evaluar su eficacia consideremos sus principales expectativas: una relación positiva entre la aplicación de la normativa y el menor número de los ACR, y el avance en la compatibilidad ACR-OMC. La gráfica 1 clasifica los ACR en función de lustros y tipos de acuerdo. ${ }^{15}$ Desde una perspectiva general, su proliferación no parece tener una relación visible con: $a$ ) el lento desgravamen NMF; $b$ ) los resultados insatisfactorios de las reuniones ministeriales de la omc, o $c$ ) la crisis global y el consecuente retroceso,

15

Estas estadísticas se basan en las notificaciones, no en el número de acuerdos reales, los cuales pueden ser notificados o no. Pueden estar inscritos dentro de normativas diferentes y de esa manera, un mismo acuerdo puede tener dos notificaciones si recubre el comercio de bienes y servicios. 
Gráfica 1. Notificaciones de los ACR según periodos (1957-2014)*

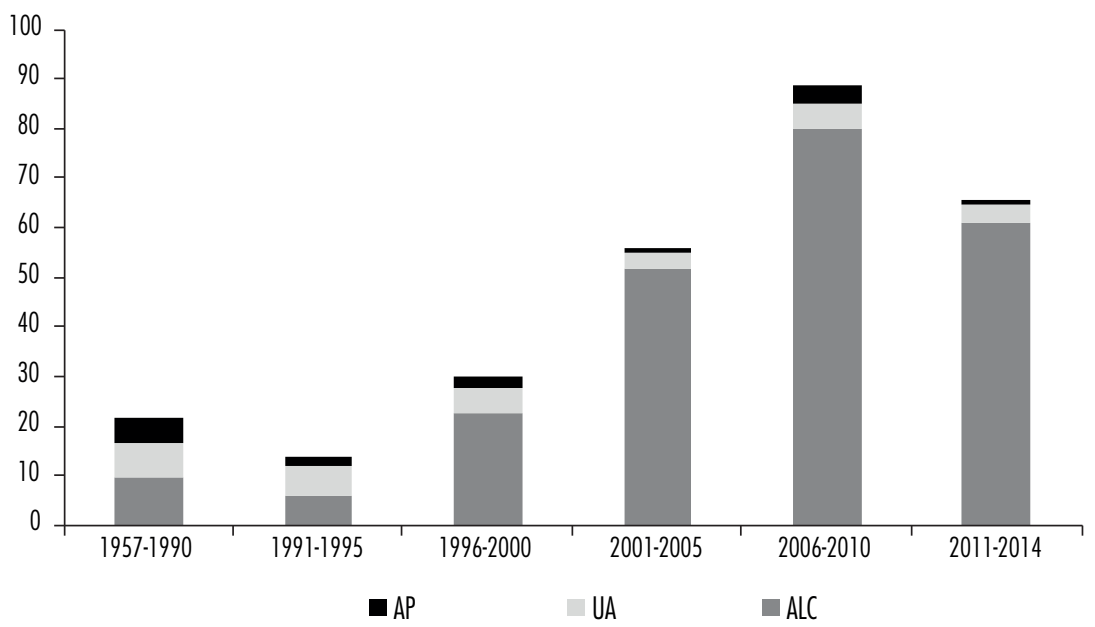

* Hasta el 3 de julio de 2014. AP: Acuerdos Preferenciales. UA: Uniones aduaneras. ALC: Áreas de Libre Comercio. Fuente: elaboración propia a partir de rtais.wto.org

estancamiento o limitado avance del comercio en los países concernidos. En el mejor de los casos estamos ante una suerte de correlación inversa entre las iniciativas regionales y las multilaterales, lo cual apoya la interpretación del regionalismo como alternativa a las insuficiencias del multilateralismo, al tiempo que refrenda la falta de "eficacia" del Art. XXIV (Davey, 2011: 242).

Pero aún no se responde a la interrogante de por qué los ACR aumentan de manera permanente. Una respuesta exhaustiva a esta interrogante escapa a los propósitos del presente artículo, aunque varios elementos ya han sido esbozados y se conectan con dos periodos diferentes.

Periodo 1. En la década de 1990, las agendas regionalistas y la multilateral llevaron a cabo sus negociaciones en tiempos cercanos e incluso simultáneamente. Esto permitió que los compromisos alcanzados en un determinado ámbito se buscaran trasladar a otras mesas. Ese fue el caso del mecanismo de solución de controversias, compuesto de resoluciones vinculantes y paneles ad hoc con cinco expertos. Bajo esa modalidad, se llevó del Tratado de Libre Comercio de América del Norte (TLCAN) a los acuerdos finales de la Ronda Uruguay, a los grupos de trabajo del ALCA y a los tratados de libre comercio de México, Canadá y Estados Unidos con países de América Latina. De esa forma, las diferentes agendas se dinamizaron entre sí, dando lugar al "efecto dominó" o de vasos comunicantes. 
Periodo 2. Después de ese periodo desaparece el ejercicio de retroalimentación entre los dos niveles sobre todo a partir de 2003. El lustro 2009-2014, enmarcado por la crisis financiera, incide aún más en la lentitud o la virtual parálisis de los objetivos de la OMC, al tiempo que los ACR continúan su despliegue con una lógica relativamente autónoma. Esa trayectoria hace que la propia toma de decisiones invoque la conjetura del regionalismo como alternativa a los problemas de la OMC (UNASUR, 2008), ilustrada recientemente por la negociación del TPP y la ATCI, cuyo énfasis en la facilitación del comercio (eliminando trabas y profundizando estándares) retoma uno de los objetivos postergados del multilateralismo.

El cambio en las condiciones internacionales en tanto que criterio analítico, lo refuerza un aspecto poco estudiado: el organizacional. En efecto, cuatro características organizacionales de los ACR creados a partir de los ańos noventa explican en parte significativa su crecimiento: 1) las dimensiones preferentemente bilaterales necesitan el consenso de pocos países; 2) se adaptan más fácilmente a los objetivos del país hub; 3) comporten a menudo una limitada cobertura temática, lo que permite negociaciones menos prolongadas; y 4) son más sensibles a las exigencias de la OMC. A todas estas razones cabe agregar otra importante: la disminuida capacidad del Art. XXIV, el instrumento por excelencia de la compatibilización.

\section{EXAMEN Y COMPATIBILIDAD}

Existe aún un otro aspecto importante por considerar: la disminuida capacidad del Art. XXIV. Vayamos por partes. En general, los ACR pueden estar en alguna de las siguientes situaciones: en proceso de negociación, firmado pero no vigente, en vigor o haber sido abandonado. De manera ideal, un nuevo acuerdo debe notificarse a la omc después de su ratificación y antes de su entrada en funciones (etapas 1, 2 y 3 en la figura 1). ${ }^{16}$ El examen (etapa 5) contiene tres subetapas que pueden caracterizarse como sigue: Presentación fáctica publicada o distribuida; Presentación fáctica no distribuida; y Presentación fáctica en suspenso.

16

El espacio que media entre la conclusión de un acuerdo y la ratificación también involucra una serie de negociaciones, sobre todo si en el proceso de ratificación alguno de los congresos nacionales modifica el acuerdo. 
Figura 1. Notificación de los ACR según etapas

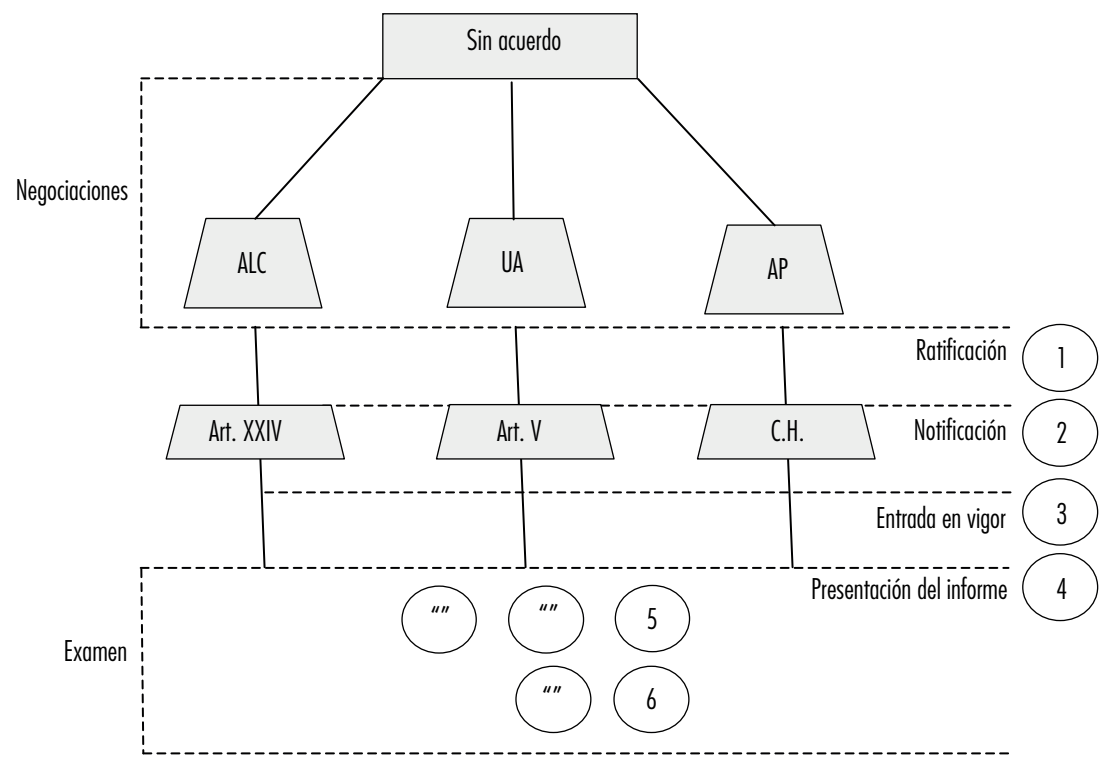

Acuerdo notificado

Fuente: elaboración propia.

La Presentación fáctica es un documento preparado por la Secretaría de la OMC en el cual se inscriben las características de los ACR, su entorno comercial y las ventajas en acceso a mercados. Su información se basa en los informes presentados por los países miembros de 10 a 20 semanas después de la notificación (etapa 4). Si la Presentación se encuentra en situación de "no distribuido" o "en suspenso", puede significar que las negociaciones todavía no han concluido o que falta completar algún compromiso importante. Dos tercios de los ACR han cumplido con este requisito amparándose en el Art. XXIV del gatT, el Art. V del gats o la Cláusula de Habilitación. El extracto fáctico (etapa 6), por su parte, es un documento de menor importancia que resume las características del acuerdo. También se prepara en la Secretaría de la omc una vez que los ACR han sido examinados. El extracto fáctico puede estar "distribuido" o "no distribuido", aunque en su mayoría ya han sido publicados (véase cuadro 2).

Durante el examen del acuerdo, el CACR monitorea el cumplimiento de los compromisos acordados internamente, recaba y sintetiza la información, y da seguimiento a los plazos de entrega de los informes de los acuerdos. En abril de 2014, estaban pendientes de la entrega del informe los responsables de 


\begin{tabular}{lrrrr}
\hline & CH & Art. V & Art. XXIV & Total \\
& & GATS & GATT & \\
\hline Presentación fáctica no distribuida & 12 & 21 & 69 & 102 \\
Presentación fáctica en suspenso & 0 & 4 & 0 & 4 \\
Presentación fáctica distribuida & 5 & 75 & 101 & 181 \\
Extracto fáctico no distribuido & 0 & 0 & 0 & 0 \\
Extracto fáctico distribuido & 11 & 21 & 40 & 72 \\
Informe adoptado & 1 & 0 & 17 & 18 \\
Total & 37 & 121 & 227 & 385 \\
\hline
\end{tabular}

Fuente: rtais.wto.org

114 acuerdos, aunque sus plazos límite son bastante desiguales. En el caso de 10 ACR, el plazo vence en 2014; de 14 en 2015, de 10 en 2016, de cinco en 2017, de tres en 2018 y de 72 en 2019 o después. El plazo más llamativo corresponde al acuerdo entre la Unión Europea y los Estados del CARIFORUM, el cual dispone de 19 años (hasta 2033). La razón de estas diferencias es que dependen de los periodos de transición de los acuerdos (omc, 2014a).

A pesar del mayor rigor del Art. XXIV y sus refuerzos, la aprobación de los ACR no presenta dificultades significativas. Las etapas 2, 4, 5 y sobre todo 6 exigen un esfuerzo administrativo comparable, por ejemplo, a una investigación anti-dumping. El elemento decisivo de la evaluación (el promedio arancelario o el arancel externo común), pierde efectividad ante dos nuevos factores. El primero, el factor contextual, impulsa la viabilidad del regionalismo hacia la renovación temática y la profundización de los compromisos a niveles mayores que el multilateralismo. El segundo, la pérdida de importancia de la protección arancelaria subraya el efecto de las barreras no arancelarias, las cuales pueden ser controladas con mayor eficacia a nivel regional. En su conjunto, estos aspectos hacen que el Art. XXIV parezca relativamente infundado y su cometido, superficial.

Sin sorpresas, las deficiencias jurídicas de esta normativa (Davey, 2011) y en general la falta de supervisión de los ACR (OMC, 2012), ha estimulado en la OMC la elaboración de recomendaciones para mejorar la "coherencia" entre el regionalismo y el multilateralismo. Uno de 
sus recientes informes sugiere cuatro grupos de iniciativas: a) reducción de los aranceles NMF para atenuar los efectos negativos de los acuerdos regionales; b) corregir las deficiencias jurídicas de la normativa; c) adoptar un enfoque flexible de tipo "legislación indicativa" como complemento de la existente; $\mathrm{y} d$ ) multilateralizar el regionalismo mediante la ampliación y fusión de los acuerdos existentes (omc, 2012: 189-191). Sin embargo, la puesta en aplicación de estas políticas representa en sí mismo un desafío, como la reducción del arancel NMF, o la ampliación y fusión de acuerdos.

\section{CONCLUSIONES}

Nuestro estudio sobre la interacción entre el regionalismo y el multilateralismo a través del Art. XXIV, arroja dos principales conclusiones. Según la primera, la proliferación de los ACR responde a una relativa autonomía de objetivos. Los rasgos organizacionales favorecen esa tendencia y explican, al menos en parte, por qué los acuerdos exceden su función de alternativa a las insuficiencias multilaterales o a la supuesta rivalidad. La segunda conclusión sostiene que el proceso de aprobación de los ACR bajo el Art. XXIV y otras normas de la OMC no inhiben las iniciativas regionales, sino que, al contrario, les otorgan una suerte de certificado de aceptación ante las instancias multilaterales. En los hechos, el examen, las etapas de la aprobación y el criterio arancelario impactan sobre aspectos relativamente superficiales de los ACR, no sobre los factores que inciden directamente sobre su creación.

Ambas conclusiones refrendan la hipótesis de que el regionalismo responde sobre todo a intereses relativamente autónomos. Los ACR en sus variantes bilaterales, plurilaterales o transregionales, son copartícipes de la creación del sistema económico mundial y su vitalidad les augura la supervivencia en un futuro cercano. Cabe precisar que esta conjetura no agota los significados del asunto tratado en el presente artículo, ni ignora la relevancia de otros aspectos. Los resultados a los que llegaría el análisis individual de los acuerdos, por ejemplo, revelarían la evidente heterogeneidad de los casos. Aunque ese tipo de investigación escapa a nuestros objetivos y excede las dimensiones del trabajo, no se excluye que sus respuestas maticen o invaliden nuestras conclusiones.

\section{AGRADECIMIENTOS}

Se agradecen las observaciones y la atenta lectura de los evaluadores anónimos. 


\section{BIBLIOGRAFÍA}

ADв (2008), Emerging Asian Regionalism. A Partnership for Shared Prosperity, Asian Development Bank, Mandaluyong.

Baldwin, R. (1995), “A Domino Theory of Regionalism”, en R. Baldwin, P. Haaparanta y J. Kiander (eds.), Expanding European Regionalism: The EU's New Members, Cambridge University Press, Cambridge.

(2010), " $21^{\text {st }}$ Century Regionalism: Filling the gap between 21 st Century Trade and 20th Century Trade Rules", Working Paper nr. 201031, Geneva Graduate Institute, Ginebra.

Baldwin, R., S. Evenett, y P. Low (2009), "Beyond Tarifs: Multilateralizing, Non-Tariff RTA Commitments", en R. Baldwin y P. Low (eds.), Multilateralizing Regionalism, Cambridge University Press, Cambridge, pp. 79-141.

Baldwin, R., y D. Jaimovich (2012), "Are Free Trade Agreements Contagious?", Journal of International Economics, vol. 88, núm. 1, 16 p.

Bhagwati, J. (1993) "Regionalism and Multilateralism: An Overview", en J. Melo y A. Panagariya (eds.), New Dimensions in Regional Integration, Cambridge University Press, Nueva York, pp. 82-87.

Bhagwati, J., y A. Panagariya (1996), "Preferential Trading Areas and Multilateralism: Strangers, Friends or Foes?”, en J. Bhagwati y A. Panagariya (eds.), The Economics of Preferential Trade Agreements, AEI Press, Washington.

Buzan, B., y O. Waever (2003), Regions and Powers, Cambridge University Press, Nueva York.

Cepal (1994), El regionalismo abierto en América Latina y el Caribe. La integración económica al servicio de la transformación productiva con equidad (LC/G.1801-SES.25/4), Santiago.

(2014), Cambio estructural para la igualdad. Una visión integrada del desarrollo (LC/G.2604), Santiago.

Chen, M. X., y A. Mattoo (2008), "Regionalism in Standards: Good or Bad for Trade?", The Canadian Journal of Economics / Revue canadienne d'Économie, vol. 41, núm. 3, pp. 838-863.

Davey, W. J. (2011), "A Model Article XXIV: are there Realistic Possibilities to Improve It?”, en K. Bagwell y P. C. Mavroidis (eds.), Preferential Trade Agreements. A Law and Economics Analysis, Cambridge University Press, Nueva York.

Fabbricotti, A. (2009), "The Paradox of Multilateralizing Regionalism through Flexibility", Proceedings of the Annual Meeting, American Society of International Law, vol. 103, pp. 119-121. 
Francois, J. (2013), Reducing Transatlantic Barriers to Trade and Investment. An Economic Assessment, Centre for Economic Policy Research, Londres, marzo.

Herz, B., y M. Wagner (2011), "Regionalism as a Building Block for Multilateralism”, Global Economy Journal, vol. 11, núm. 1, p. 29.

Hettne, B., y F. Söderbaum (2000), "Theorising the Rise of Regionness", New Political Economy, vol. 5, núm. 3, pp. 457-472.

Hoogensen, G. (2005), "Bottoms Up! A Toast To Regional Security?", International Studies Review, vol. 7, núm. 2, pp. 269-274.

Kerry (2007), "Security Theory in the New Regionalism", International Studies Review, vol. 9, núm. 2, pp. 197-229.

Kono, D. Y. (2007), "When do Trade Blocs Block Trade?”, International Studies Quarterly, vol. 51, núm. 1, pp. 165-181.

Kowalczk, C., y R. J. Wonnacott (1992), "Hub and Spokes, and Free Trade in the Americas", Working Paper núm. 92-14, Department of Economics, Dartmonth College, Hanover.

Krugman, P. (1993), "Regionalism versus Multilateralism: Analytic Notes", en J. de Melo y A. Panagariya (eds.), New Dimensions in Regional Integration, Cambridge University Press, Cambridge.

Lee, Y.-S. (2011), "Reconciling RTAS with the wTo Multilateral Trading System: Case for a New Sunset Requirement on RTAS and Development Facilitation”, Journal of World Trade, vol. 45, núm. 3, pp. 629-651.

Lipsey, R. G. y M. G. Smith (1989), "The Canada-US Free Trade Agreement: Special Case or Wave of the Future?”, en J.J. Schott, Free Areas and U.S. Trade Policy, Institute for International Economics, Washington D. C.

López, C., y A. y J. Soler (1998), "Open Regionalism versus Discriminatory Trading Agreements", ASEAN Economic Bulletin, vol. 14, núm. 3, pp. 4562.

Marconini, M. (2010), "Los servicios en los acuerdos regionales entre países de América Latina y países desarrollados”, Temas controversiales en negociaciones comerciales Norte-Sur, Comisión Económica para América Latina y el Caribe, Santiago, agosto, pp. 59-96.

Michalak, W., y R. Gibb (1997), "Trading Blocs and Multilateralism in the World Economy", Annals of the Association of American Geographers, vol. 87, núm. 2, pp. 264-279.

Mistry, P. S. (1995), "Open Regionalism: Stepping Stone or Millstone Toward and Improved Multilateral System?”, en J. J. Teunissen (ed.), Regionalism and the Global Economy: The Case of Latin America and the Caribbean, FONDAD, La Haya. 
Mundell, R. A. (1964), "Tariff Preferences and The Terms of Trade", The Manchester School of Economic and Social Studies, vol. 32.

Nomura, R., T. Ohkawa, M. Okamura, y M. Tawada (2013), "Does a Bilateral FTA Pave the Way for Multilateral Free Trade?", Review of International Economics, vol. 21, núm. 1, pp. 164-176.

Nordstrom, H. (1995), "Customs Unions, Regional Trading Blocks and Welfare", R. Baldwin, P. Haaparanta y J. Kiander (eds.), Expanding Membership of the European Union, Cambridge University Press, Cambridge.

omc (1996), "Comité de Acuerdos Comerciales Regionales. Decisión de 6 de febrero de 1996”, WT/L/127 (96-0473), Ginebra, 7 de febrero.

(2001), "Concluyen con éxito en la omc las negociaciones para la adhesión de China", Comunicado de prensa, PRESS/243, Ginebra, 17 de septiembre. http:// www.wto.org/spanish/news_s/pres01_s/pr243_s.htm

(2012), Informe sobre el Comercio Mundial 2011. La OMCy los acuerdos comerciales preferenciales: de la coexistencia a la coherencia, Ginebra. http:// www.wto.org/ spanish/res_s/booksp_s/anrep_/world_trade_report_s.pdf Consultado el 4 de diciembre de 2014.

(2013), "Nota informativa: acuerdos comerciales regionales", Novena Conferencia Ministerial de la OMC, Bali, 3-7 de diciembre.

(2014a), "Comité de Acuerdos Comerciales Regionales. Lista de ACR sujetos a la presentación de los informes sobre la aplicación”, WT/ REG/W/82 (14-2525), Ginebra, 25 de abril.

(2014b), "Comité de Acuerdos Comerciales Regionales. Lista de ACR que se han mencionado en las presentaciones fácticas", WT/REG/W/83 (14-3452), Ginebra, 13 de junio.

OMC, ITC y UNCTAD (2013), Perfiles arancelarios en el mundo 2013, http:// www. wto.org/spanish/res_s/booksp_s/tariff_profiles13_s.pdf. Consultado el 5 de octubre de 2014.

Pant, M., y A. Sadhukhan (2009), "Does Regionalism Hinder Multilateralism: a Case Study of India”, Journal of Economic Integration, vol. 24, núm. 2, pp. 222-247.

Pempel, T. J. (2008), "China and the Emerging Asian Regionalism", Conference on China, FUNAG e IPRI, Río de Janeiro, 17-18 de abril, pp. 1-20.

Schiff, M. (1997), "Sall is Beautiful: Preferential Trade Agreements and the Impact of Country Size, Market Share, and Smuggling", Journal of Economic Integration, vol. 3, núm. 12, pp. 359-87.

Schiff, M., y L. A. Winters (2004), Integración regional y desarrollo, Banco Mundial y Alfaomega, Bogotá. 
Germán A. de la Reza

unasur (2008), Tratado Constitutivo de la Unión de Naciones Suramericanas, Brasilia, 23 de mayo.

Viner, J. (1950), Customs Union Issue, Carnegie Endowment, Washington, D.C.

Wilcox, C. (1949), A Chart for World Trade, McMillan, Nueva York. 